\title{
Effets Des Technologies Membranaires Sur Les Caracteristiques Physicochimiques Des Extraits De Fruits De La Passion (Passiflora Edulis)
}

\author{
Casimir Anauma Koko, Dr. \\ Benjamin Kan Kouamé, Dr. \\ Massé Diomandé, Dr. \\ Christiane Eunice Adjoua Boko, MA
}

Université Jean Lorougnon Guédé, Département de Biochimie et Microbiologie, Laboratoire d'Agrovalorisation, Côte d'Ivoire

Félix Adjé, Dr.

Emmanuel Nogbou Assidjo, Prof

Institut National Polytechnique Félix Houphouët-Boigny, Laboratoire des

Procédés Industriels de Synthèse et Environnement, Côte d'Ivoire

\section{Georges N'guessan Amani, Prof}

Université Nangui Abrogoua, UFR des Sciences et Technologies des Aliments, Laboratoire de Biochimie Alimentaire et de Technologie des

Produits Tropicaux, Côte d'Ivoire

Doi: 10.19044/esj.2018.v14n33p281 URL:http://dx.doi.org/10.19044/esj.2018.v14n33p281

\begin{abstract}
The passion fruit or grenadilla is usually consumed in the form of juice and its pleasant intensive aroma and flavor make it an attractive element for the food industry. The objective of the present work is to determine the effects of membrane technologies such as tangential microfiltration and reverse osmosis on the physicochemical characteristics of passion fruit extracts. The characteristics of the various extracts were determined according to conventional methods. In addition, statistical methods were applied to the data collected in order to determine the effects of membrane technologies. The results showed a yield of $89.33 \%$ for the extraction of the microfiltered juice and a volume reduction factor of 16.75 for the concentrate. In addition, the microfiltration-concentration process significantly $(\mathrm{p}<0.05)$ influenced the physicochemical parameters of the extracts produced. Thus, tangential microfiltration made it possible to clarify the crude extract by changing from a turbidity of $89.23 \pm 1.71$ to $0.72 \pm 0.02$ NTU. It has also resulted in a significant decrease in $\mathrm{pH}$ and flavonoids contents. Principal component analysis confirmed the existence of differences between the extracts produced
\end{abstract}


despite the various correlations between their characteristics. It revealed that the JB and CMFT extracts are characterized by their high $\mathrm{pH}$ and low density. On the other hand, the COI extract is characterized by its brix degree, viscosity, conductivity and high vitamin $\mathrm{C}$ content. In addition, reverse osmosis has the advantage of concentrating the compounds of interest of clarified juice with a concentration factor ranging from 2.15 to 9.39 .

Keywords: Passiflora edulis, tangential microfiltration, reverse osmosis, extract, Côte d'Ivoire

\section{Résumé}

Le fruit de la passion ou grenadille est généralement consommé sous forme de jus et son agréable arôme intensif et sa saveur en font un élément attractif pour les industries alimentaires. L'objectif du présent travail est de déterminer les effets des technologies membranaires telles que la microfiltration tangentielle et l'osmose inverse sur les caractéristiques physicochimiques des extraits de fruits de la passion. Les caractéristiques des différents extraits ont été déterminées selon des méthodes conventionnelles. Par ailleurs, des méthodes statistiques ont été appliquées sur les données collectées aux fins de déterminer les effets des technologies membranaires. Les résultats ont révélé un rendement de $89,33 \%$ pour l'extraction du jus microfiltré et un facteur de réduction volumique de 16,75 pour le concentré. En outre, le procédé microfiltration-concentration influence significativement $(\mathrm{p}<0,05)$ les paramètres physicochimiques des extraits produits. Ainsi, la microfiltration tangentielle a permis de clarifier l'extrait brut en passant d'une turbidité de 89,23 \pm 1,71 à 0,72 \pm 0,02 NTU. Elle a entrainé également une diminution significative du $\mathrm{pH}$ et des flavonoïdes. L'analyse en composantes principales a confirmé l'existence de différence entre les extraits produits malgré les nombreuses corrélations entre leurs caractéristiques. Elle a révélé que les extraits JB et CMFT sont caractérisés par leur pH élevé et leur faible densité. Par contre, l'extrait COI est caractérisé par son degré brix, sa viscosité, sa conductivité et sa teneur en vitamine $\mathrm{C}$ élevée. En addition, l'osmose inverse a présenté l'avantage de concentrer les composés d'intérêt du jus clarifié avec un facteur de concentration variant de 2,15 à 9,39.

Mots clés: Passiflora edulis, microfiltration tangentielle, osmose inverse, extrait, Côte d'Ivoire

\section{Introduction}

Originaire de l'Amérique du Sud, la grenadille (Passiflora edulis) est une plante grimpante de la famille des Passifloraceae (Hoff et Cremers, 2005). Elle est surtout cultivée pour ses fruits à pulpe comestible nommés fruits de la 
passion. le fruit est commercialisé dans le monde entier avec le Brésil, l'Équateur et la Colombie comme les plus grands producteurs (Cardoso et al., 2012). Il est riche en vitamines et micronutriments et lui a été associé par ailleurs, de nombreuses vertus entre-autres le soulagement des problèmes mineurs du sommeil, d'anxiété et un pouvoir antispasmodique (Winston et al., 2000 ; Barnes et al., 2002). Le fruit de la passion est généralement consommé sous forme de jus et son agréable arôme intensif et sa saveur en font un élément attractif pour les industries alimentaires (Domingues et al., 2012). De nos jours, il existe une tendance croissante de consommation de fruits tropicaux, de jus et de boissons aux fruits, en raison de l'intérêt des consommateurs pour les produits sains qui sont pratiques et prêts à être consommés (Cardoso et al., 2012).

Les méthodes traditionnelles de stabilisation, telles que la pasteurisation thermique, sont généralement appliquées pour le traitement des jus de fruits. Cependant, le traitement thermique entraîne une modification de l'arôme et de la saveur des fruits de la passion (Domingues et al., 2014). De plus, l'addition d'un conservateur chimique tel que l'anhydride sulfureux ou l'acide benzoïque, ou un mélange des deux, ne donne que des résultats médiocres notamment en ce qui concerne l'arôme et le goût (Haendler, 1965). Par conséquent, le développement et l'étude des nouvelles technologies pour obtenir des produits de haute qualité du fruit est essentiel pour encourager sa production (Cardoso et al., 2012).

Ainsi, les procédés membranaires de séparation qui ne génèrent pas de déchets, n'utilisent pas de conservateurs chimiques et adaptés au traitement des jus de fruits organiques peuvent être utilisés (Silva et al., 2005). Parmi ceux-ci, la microfiltration $(\mathrm{MF})$ est un procédé de stabilisation à froid des jus de fruits et permet de préserver les qualités nutritionnelles et organoleptiques du produit. Le choix de ce procédé se justifie également par le fait que les jus de passion sont riches en vitamines et autres nutriments thermosensibles. Dans l'optique d'améliorer la stabilité des extraits de fruits, un couplage a été initié avec l'osmose inverse. Ce dernier est utilisé dans les industries agroalimentaires pour pré-concentrer des solutions thermosensibles (Soro, 2012). De nombreux auteurs ont associé à la microfiltration, l'osmose inverse pour améliorer la stabilité de produits (Soro, 2012 ; Adjé et al., 2013 ; Cissé et al., 2014). Pour la production d'extraits de fruits de la passion, cette technologie a très peu été utilisée en Côte d'Ivoire. De ce fait, les effets d'une telle technologie sur les caractéristiques physicochimiques des extraits produits restent à déterminer.

La présente étude a eu pour objectif de déterminer l'influence des technologies membranaires telles que la microfiltration tangentielle et l'osmose inverse sur la composition physicochimique des extraits de fruits de la passion. Les objectifs spécifiques sont de : 
- produire des extraits bruts, microfiltrés et concentrés à base de fruits de passion et de caractériser leur nature physicochimique ;

- déterminer l'impact de la microfiltration et de l'osmose inverse sur les caractéristiques de ces extraits.

\section{Matériel et méthodes}

\section{Matériel biologique}

Des fruits de la passion jaune de l'espèce Passiflora edulis (var) Flavicarpa ont été utilisés (Figure 1).

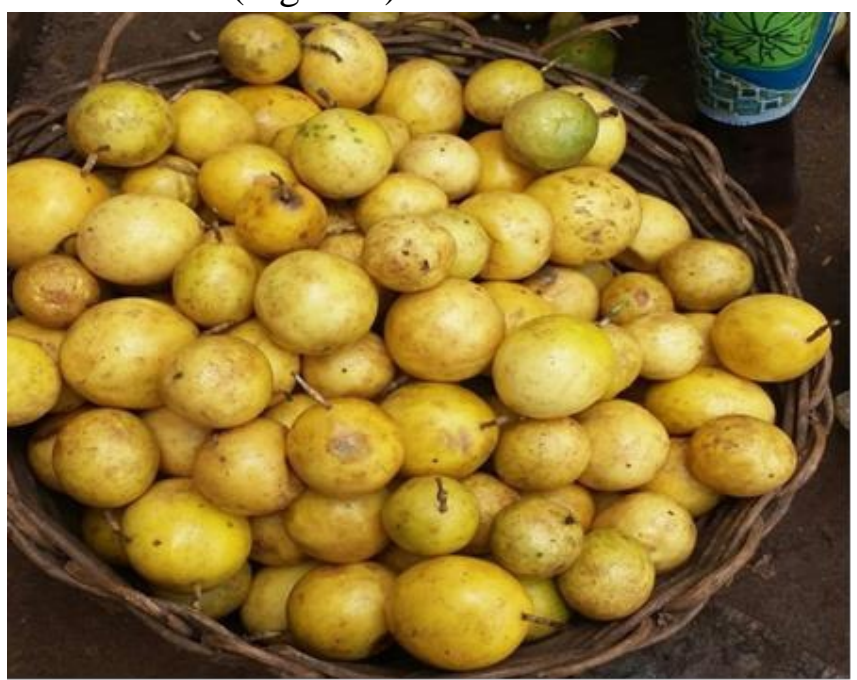

Figure 1 : Fruits de la passion jaune (Boko, 2018)

\section{Préparation des fruits de passion et production du jus brut}

Les fruits de la passion utilisés ont été achetés sur les différents marchés de la ville de Daloa (Région du Haut-Sassandra, Côte d'Ivoire). Ces fruits ont été lavés avec de l'eau courante afin d'éliminer les salissures et les impuretés. Ils ont été coupés en deux à l'aide d'un couteau en acier inoxydable afin de retirer la pulpe. Une fois la pulpe obtenue $(25 \mathrm{~kg})$, l'extrait brut a été préparé en faisant une dilution $1: 5$ (pulpe : eau) puis une pré-filtration avec un tamis de $40 \mathrm{~mm}$.

\section{Production des extraits par microfiltration tangentielle (MFT) et par osmose inverse (OI)}

La production des extraits a été faite selon la méthode décrite par Adjé et al. (2013) comprenant une procédure de lavage du pilote et la filtrationconcentration proprement dite. La clarification a été réalisée par microfiltration tangentielle grâce à un pilote semi industriel de marque TIA (Bollène, France) d'une capacité de 50 L (Figure 2). L'extrait brut a été placé 
dans le réservoir d'alimentation et distribué dans l'unité de microfiltration. Le flux d'alimentation a été pompé à partir d'une température réservoir de contrôleur $\left(25{ }^{\circ} \mathrm{C}\right)$ à travers la membrane, avec 1 bar pour la pompe d'alimentation et 2,2 bars pour la pompe de circulation. L'extrait microfiltré a été recueilli dans le bac d'osmose inverse. Pour la concentration, la pression transmembranaire imposée a été de 40 bars. L'extrait issu de la microfiltration est concentré jusqu'au maximum de réduction de son volume. Le volume du concentré final ainsi récupéré correspond à l'extrait concentré.

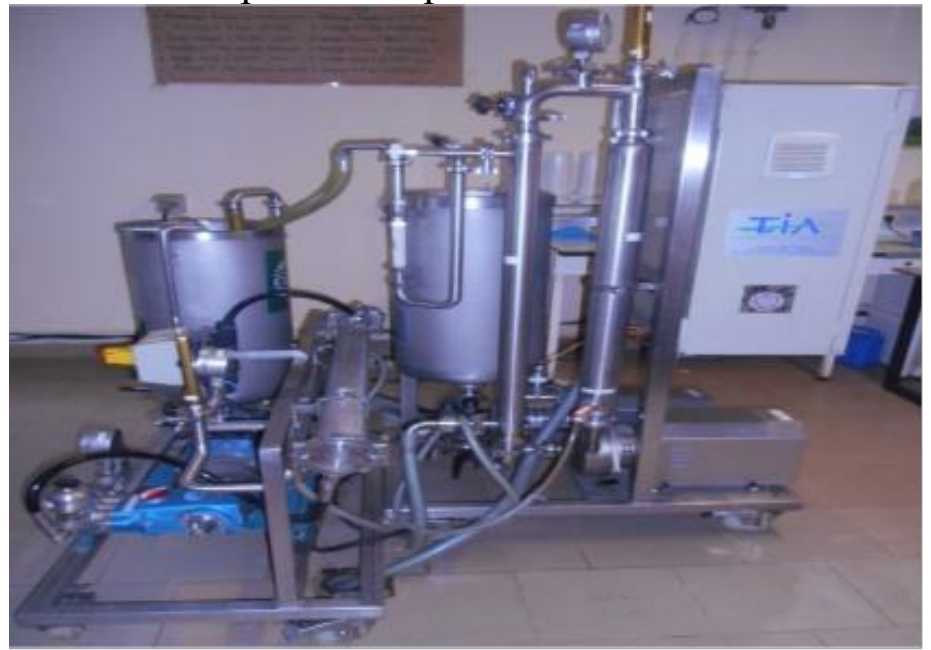

Figure 2 : Pilote semi industriel de marque TIA (Boko, 2018).

\section{Détermination des facteurs de réduction volumique (FRV) et de concentration (FC)}

Le volume total de perméat d'OI a servi à calculer le facteur de réduction volumique à tout instant $\mathrm{t}(\mathrm{FRVt})$ de l'extrait microfiltré en cours de concentration, selon la formule décrite par Adjé et al. (2013) :

$\mathrm{FRV}_{\mathrm{t}}=(\mathrm{Vi} / \mathrm{Vc})=(\mathrm{Vi} /(\mathrm{Vi}-\mathrm{Vw}))$

avec Vi : volume initial de perméat de MFT utilisé ;

$\mathrm{Vw}$ : volume de perméat d'OI au temps $\mathrm{t}$;

$\mathrm{Vc}$ : volume calculé d'extrait concentré au temps t.

Le facteur de concentration (FC) des composés extraits est déterminé par la relation suivante :

$\mathrm{FC}=\mathrm{CCOI} / \mathrm{CEMFT}$

avec CCOI : Concentration du composé dans l'extrait concentré ; CEMFT : Concentration du composé dans l'extrait microfiltré. 


\section{Analyses physicochimiques des extraits de fruits de passion}

Des caractéristiques physicochimiques ont été déterminées sur les extraits de fruits de la passion présentés sur la figure 3. Le $\mathrm{pH}$ a été déterminé selon la méthode AOAC (2000). L'acidité titrable a été déterminée par titrage au moyen d'une solution d'hydroxyde de Sodium $(0,1 \mathrm{~N})$ en présence de phénolphtaléine. L'extrait sec soluble (ESS) a été mesuré à l'aide un réfractomètre (Leica AR 200) en mettant 3 à 4 gouttes d'extrait de fruits de la passion sur le prisme après étalonnage à l'aide de l'eau distillée. La turbidité des extraits de fruits de la passion a été déterminée selon la méthode décrite par Araya-Farias (1999). La conductivité des extraits du fruit de la passion a été quantifiée à l'aide d'un conductimètre (Hanna HI 9835) en plongeant l'électrode dans une prise d'essai de $50 \mathrm{~mL}$. La teneur en vitamine C a été dosée selon la méthode décrite par Elgamouz (2016). La viscosité des extraits de fruits de la passion a été déterminée selon la méthode décrite par Boutarfa (2012) à l'aide d'un viscosimètre à chute de bille de type Thermo Scientific. La méthode décrite par Wood et al. (2002) a été utilisée pour le dosage des polyphénols totaux. La concentration en polyphénols a été exprimée en milligramme par litre d'extrait équivalent acide gallique (mg.L ${ }^{-1}$, Eq.AG). Par ailleurs, le dosage des flavonoïdes a été réalisé selon la méthode de Marinova et al. (2005) et la teneur a été exprimée en milligramme par litre d'extrait équivalent quercétine (mg.L $\left.{ }^{-1} \mathrm{Eq} . \mathrm{Q}\right)$.

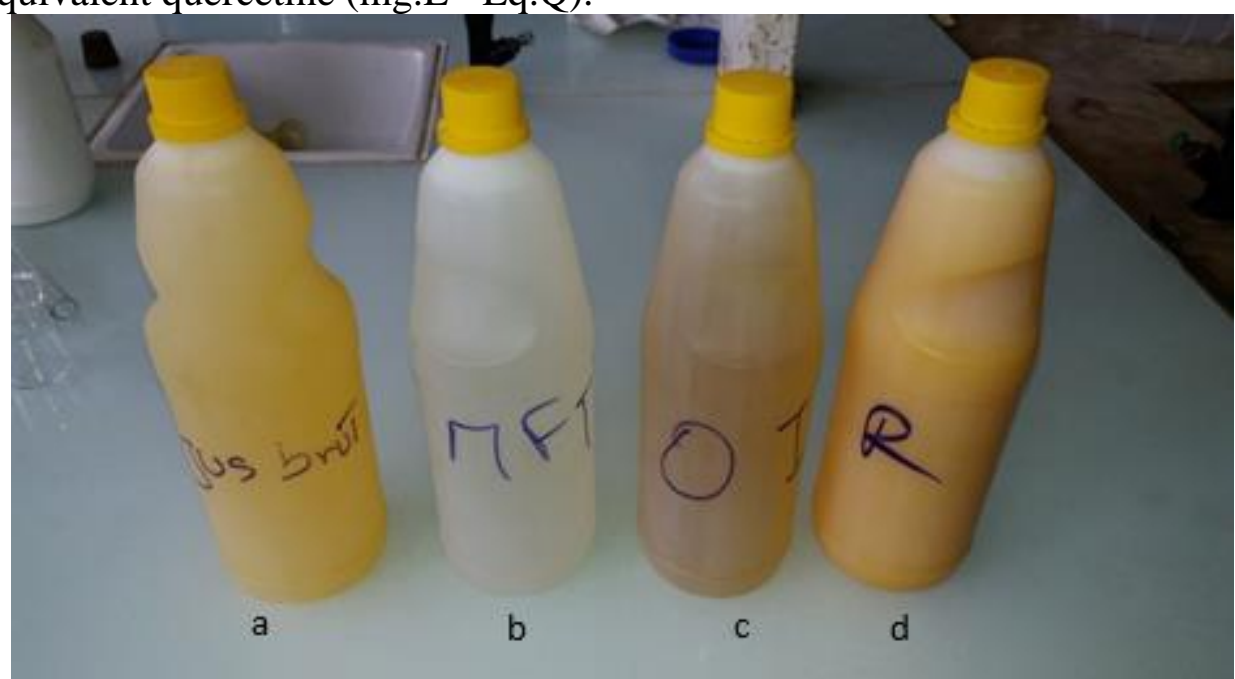

Figure 3 : Différents extraits de fruits de la passion (a : Extrait brut ; b : Extrait microfiltré ; c : Extrait concentré ; d : Retentât) (Boko, 2018)

\section{Analyses statistiques}

Les analyses statistiques ont été effectuées sur les données recueillies de la caractérisation physicochimique des extraits de fruits de la passion. Une analyse de variance multidimensionnelle a été réalisée aux fins d'apprécier 
l'existence de différence entre les échantillons étudiés. Par ailleurs, des analyses de variance ont été également effectuées sur ces données. Des tests de comparaison multiples (Tukey HSD) ont été conduits lorsque la différence a été révélée comme significative $(\mathrm{p}<0,05)$ aux fins de séparer les différents extraits. Enfin, l'analyse en composantes principales a été effectuée aux fins de visualiser les différences et d'associer aux différents groupes identifiés, leurs caractéristiques principales. Pour ces traitements statistiques, le logiciel STATISTICA 7.1 a été utilisé.

\section{Résultats}

\section{Production des extraits de fruits de la passion}

A partir de $150 \mathrm{~L}$ de jus brut, la production d'extrait de passion a été réalisée au pilote semi-industriel et a fourni 134 L de jus microfiltré (CMFT) soit un rendement de $89,33 \%$. La concentration de ce jus CMFT a abouti à un facteur de réduction volumique (FRV) de 16,75 pour l'extrait COI (Tableau 1).

Tableau 1 : Conditions opératoires de production des extraits de fruits de passion

$\begin{array}{cccccc}\begin{array}{c}\text { Ratio } \\ (\mathrm{v} / \mathrm{v})\end{array} & \begin{array}{c}\text { Volume } \\ \text { d'alimentation } \\ \text { (VA) en L }\end{array} & \begin{array}{c}\text { CMFT } \\ (\text { en L) }\end{array} & \begin{array}{c}\text { Retentât } \\ (\text { en L) }\end{array} & \begin{array}{c}\text { COI } \\ (\text { en L) }\end{array} & \text { FRV } \\ & 150 & & & \end{array}$

\begin{tabular}{|c|c|c|c|c|c|c|}
\hline Production & $25 / 150$ & 150 & 134 & 16 & 8 & 16,75 \\
\hline
\end{tabular}

\section{Caractéristiques physicochimiques des extraits de fruits de la passion}

Les différents extraits de fruits de la passion présentent un $\mathrm{pH}$ acide avec des valeurs variant de 4,36 $\pm 0,23(\mathrm{JB})$ à 2,87 $\pm 0,01(\mathrm{COI})$ en passant par une valeur de 3,30 $\pm 0,10$ pour l'extrait CMFT et de 3,03 $\pm 0,05$ pour le retentât (Tableau 2). L'analyse comparée des extraits JB et CMFT montre une diminution significative de la turbidité allant de 89,23 $\pm 1,71$ NTU à 0,72 \pm 0,02 NTU. Cette diminution est aussi observée au niveau de la teneur en flavonoïdes contrairement à la vitamine $\mathrm{C}$ et la conductivité qui augmentent significativement. En outre, la densité, l'acidité titrable, l'ESS, la viscosité et la teneur en polyphénols de ces deux extraits ne présentent pas de différence significative $(\mathrm{p}<0,05)$. Par ailleurs, l'extrait concentré présente des valeurs nutritionnelles significativement supérieures à celles du microfiltré et du jus brut, avec des facteurs de concentration variant de 2,15 (pour la vitamine $\mathrm{C}$ ) à 9,39 (pour l'ESS). De plus, cet extrait a donné les valeurs les plus élevées pour la densité $(1,65 \pm 0,01)$ la conductivité $(5928,33 \pm 2,89 \mathrm{mS})$, l'acidité titrable $\left(618 \pm 14\right.$ meq. $\left.\mathrm{L}^{-1}\right)$ et la viscosité $(1,67 \pm 0,01 \mathrm{mPa} . \mathrm{s})$. Au niveau des caractéristiques physicochimiques telles que les flavonoïdes et les polyphénols totaux, c'est le retentât qui a enregistré les valeurs les plus élevées avec 
respectivement $5,63 \pm 0,41 \mathrm{mg} / \mathrm{L}$ Eq.Q et $4,47 \pm 0,03 \mathrm{mg} / \mathrm{L}$ Eq.AG. $\quad \mathrm{Ce}$ retentât renferme en outre, de la vitamine C $(793 \pm 1,73 \mathrm{mg} / \mathrm{L})$.

Tableau 2 : Caractéristiques physicochimiques des extraits de fruits de la passion

Extraits de fruits de la passion

\begin{tabular}{|c|c|c|c|c|c|}
\hline Paramètres & JB & CMFT & RT & COI & FC \\
\hline $\mathrm{pH}\left(25^{\circ} \mathrm{C}\right)$ & $4,36 \pm 0,23^{\mathrm{a}}$ & $3,30 \pm 0,10^{\mathrm{b}}$ & $3,03 \pm 0,05^{\mathrm{c}}$ & $2,87 \pm 0,01^{\mathrm{d}}$ & - \\
\hline Densité & $1,43 \pm 0,01^{\mathrm{a}}$ & $1,42 \pm 0,01^{\mathrm{a}}$ & $1,51 \pm 0,01^{\mathrm{b}}$ & $1,65 \pm 0,01^{\mathrm{c}}$ & - \\
\hline $\begin{array}{l}\text { Acidité titrable } \\
\left(\text { meq. } \mathrm{L}^{-1}\right)\end{array}$ & $44,67 \pm 4,16^{\mathrm{a}}$ & $54,67 \pm 4,16^{\mathrm{a}}$ & $75,33 \pm 5,03^{b}$ & $618 \pm 14^{c}$ & - \\
\hline Turbidité (NTU) & $89,23 \pm 1,71^{\mathrm{a}}$ & $0,72 \pm 0,02^{\mathrm{b}}$ & $886,33 \pm 2,3^{\mathrm{c}}$ & $29,53 \pm 0,37^{b}$ & - \\
\hline ESS $\left({ }^{\circ}\right.$ Brix $)$ & $1,63 \pm 0,15^{\mathrm{a}}$ & $2,03 \pm 0,05^{\mathrm{a}}$ & $1,8 \pm 0,00^{\mathrm{b}}$ & $19,07 \pm 0,11^{\mathrm{c}}$ & 9,39 \\
\hline $\begin{array}{l}\text { Viscosité } \\
\text { (mPa.s) }\end{array}$ & $1,09 \pm 0,00^{\mathrm{a}}$ & $1,09 \pm 0,01^{\mathrm{a}}$ & $1,13 \pm 0,02^{\mathrm{a}}$ & $1,67 \pm 0,01^{\mathrm{b}}$ & - \\
\hline $\begin{array}{l}\text { Vitamine } \\
(\mathrm{mg} / \mathrm{L})\end{array}$ & $653,66 \pm 14,57^{\mathrm{a}}$ & $752 \pm 6,92^{b}$ & $793 \pm 1,73^{c}$ & $1619 \pm 9^{d}$ & 2,15 \\
\hline $\begin{array}{l}\text { Flavonoïdes } \\
(\mathrm{mg} / \mathrm{L} \text { Eq.Q) }\end{array}$ & $1,79 \pm 0,06^{\mathrm{a}}$ & $0,62 \pm 0,19^{b}$ & $5,63 \pm 0,41^{\mathrm{c}}$ & $2,05 \pm 0,50^{\mathrm{a}}$ & 3,30 \\
\hline $\begin{array}{l}\text { Polyphénols } \\
\text { (mg/L Eq.AG) }\end{array}$ & $0,59 \pm 0,00^{\mathrm{a}}$ & $0,21 \pm 0,06^{\mathrm{a}}$ & $4,47 \pm 0,03^{\mathrm{b}}$ & $1,65 \pm 2,24^{\mathrm{c}}$ & 7,85 \\
\hline $\begin{array}{l}\text { Conductivité } \\
(\mathrm{mS})\end{array}$ & $\begin{array}{c}1203,67 \pm \\
2,08^{\mathrm{a}} \\
\end{array}$ & $1514,33 \pm 4,93^{\mathrm{b}}$ & $1471,33 \pm 2,30^{c}$ & $\begin{array}{l}5928,33 \pm \\
2,89^{\mathrm{d}}\end{array}$ & - \\
\hline
\end{tabular}

Les valeurs avec des lettres alphabétiques différentes sur la même ligne sont statistiquement différentes $(p<0,05)$; JB : Jus Brut ; CMFT : Jus Clarifié par Microfiltration Tangentielle ;

COI : Jus Concentré par Osmose Inverse ; RT : Retentât ; FC : Facteur de concentration

\section{Effets de la technologie membranaire sur l'ensemble des paramètres physicochimiques}

L'analyse des effets de la technologie membranaire sur l'ensemble des paramètres physico chimiques a montré qu'il existe une différence significative $(p<0,05)$ entre les différents extraits de passion (Tableau 3). Par ailleurs, de nombreuses relations significatives et positives existent entre les paramètres physicochimiques des extraits de passion (Tableau 4). C'est le cas entre l'acidité et des paramètres tels que la viscosité (coefficient $=0,99)$, la conductivité (coefficient $=1$ ), la teneur en vitamine $\mathrm{C}$ (coefficient $=1$ ), les polyphénols $(0,99)$ et la densité $(0,93)$. Il en va de même entre l'ESS et les paramètres comme la viscosité (coefficient $=0,99$ ), la conductivité (coefficient $=1$ ), la vitamine $\mathrm{C}$ (coefficient $=0,99$ ), les polyphénols (coefficient $=0,99$ ) et la densité (coefficient $=0,92$ ). De plus, la turbidité est corrélée positivement avec la teneur en flavonoïdes (coefficient $=0,96$ ).

Il existe aussi une corrélation significative entre la viscosité et les paramètres comme la conductivité (coefficient $=0,99$ ), la vitamine $\mathrm{C}$ (coefficient $=0,99$ ), les polyphénols (coefficient $=0,99$ ) et la densité 
(coefficient $=0,94)$. De telles corrélations existent entre la conductivité et les paramètres comme la vitamine $\mathrm{C}$ (coefficient $=1$ ), les polyphénols (coefficient $=0,99$ ) et la densité (coefficient $=0,92$ ). De plus, la vitamine $C$ est corrélée avec les polyphénols (coefficient $=0,99$ ) d'une part et avec la densité (coefficient $=0,95$ ) d'autre part. Enfin, une corrélation significative existe entre les polyphénols et la densité (coefficient $=0,96$ ).

En définitive, les corrélations les plus significatives sont entre l'acidité et le degré brix, entre l'acidité et la conductivité, entre l'acidité et la vitamine $\mathrm{C}$, entre le degré brix et la conductivité et entre la vitamine $\mathrm{C}$ et la conductivité.

Tableau 3 : Résultat de l'analyse de variance multidimensionnelle

\section{Effets de la technologie membranaire sur chacun des paramètres physicochimiques des extraits.}

Les résultats montrent que la technologie membranaire a un effet significatif $(\mathrm{p}<0,05)$ sur chacun des paramètres physicochimiques des différents extraits de passion (Tableau 5). 
Tableau 4IV : Corrélation entre les variables caractérisant les extraits de fruits de la passion

\begin{tabular}{|c|c|c|c|c|c|c|c|c|c|c|}
\hline & \multirow{2}{*}{\multicolumn{2}{|c|}{ Acidité }} & \multirow[b]{2}{*}{ ESS } & \multirow[b]{2}{*}{ Turbidité } & & & & & & \\
\hline & & & & & Viscosité & Conductivité & \multicolumn{2}{|r|}{ Flavonoïdes } & Polyphénols & Densité \\
\hline $\mathrm{pH}$ & 1,00 & & & & & & & & & \\
\hline Acidité & $-0,48$ & 1,00 & & & & & & & & \\
\hline ESS & $-0,46$ & 1,00 & 1,00 & & & & & & & \\
\hline Turbidité & $-0,21$ & $-0,31$ & $-0,35$ & 1,00 & & & & & & \\
\hline Viscosité & $-0,48$ & 0,99 & 0,99 & $-0,28$ & 1,00 & & & & & \\
\hline Conductivité & -0.50 & 1,00 & 1,00 & $-0,33$ & 0,99 & 1,00 & & & & \\
\hline Vitamine $\mathrm{C}$ & -0.56 & 1,00 & 0,99 & $-0,26$ & 0,99 & 1,00 & 1,00 & & & \\
\hline Flavonoïdes & $-0,21$ & $-0,10$ & $-0,15$ & 0,96 & $-0,07$ & $-0,13$ & $-0,07$ & 1,00 & & \\
\hline Polyphénols & $-0,49$ & 0,99 & 0,99 & $-0,22$ & 0,99 & 0,99 & 0,99 & $-0,02$ & 1,00 & \\
\hline Densité & $-0,57$ & 0,93 & 0,92 & 0,05 & 0,94 & 0,92 & 0,95 & 0,25 & 0,96 & 1,00 \\
\hline
\end{tabular}


Tableau 5 : Effets de la technologie membranaire sur chacun des paramètres physicochimiques des extraits de fruits de la passion

\begin{tabular}{|c|c|c|c|c|c|}
\hline Paramètres & Effet & $\mathrm{SC}$ & $\mathrm{MC}$ & $\mathrm{F}$ & $\mathrm{P}$ \\
\hline \multirow[t]{2}{*}{$\mathrm{pH}$} & Technologie & 2,6075 & 0,8692 & 4345,8 & 0,00 \\
\hline & Erreur & 0,0016 & 0,0002 & & \\
\hline \multirow[t]{2}{*}{ Acidité } & Technologie & 706507,7 & 235502,6 & 3679,72 & 0,00 \\
\hline & Erreur & 512,0 & 64,0 & & \\
\hline \multirow[t]{2}{*}{ ESS } & Technologie & 669,3267 & 223,1089 & 22310,89 & 0,00 \\
\hline & Erreur & 0,0800 & 0,0100 & & \\
\hline \multirow[t]{2}{*}{ Turbidité } & Technologie & 1624506 & 541502,0 & 257220,1 & 0,00 \\
\hline & Erreur & 17 & 2,1 & & \\
\hline \multirow[t]{2}{*}{ Viscosité } & Technologie & 0,716112 & 0,23871 & 306,57 & 0,00 \\
\hline & Erreur & 0,00623 & 0,00078 & & \\
\hline \multirow[t]{2}{*}{ Conductivité } & Technologie & 46380546 & 15460182 & 1460805 & 0,00 \\
\hline & Erreur & 85 & 11 & & \\
\hline \multirow[t]{2}{*}{ Vitamine $\mathrm{C}$} & Technologie & 0,000058 & 0,000019 & 5965,1 & 0,00 \\
\hline & Erreur & 0,000000 & 0,000000 & & \\
\hline \multirow[t]{2}{*}{ Flavonoïdes } & Technologie & 42,08466 & 14,02822 & 119,973 & 0,00 \\
\hline & Erreur & 0,93542 & 0,11693 & & \\
\hline \multirow[t]{2}{*}{ Polyphénols } & Technologie & 2007,208 & 669,069 & 532,4654 & 0,00 \\
\hline & Erreur & 10,052 & 1,257 & & \\
\hline \multirow[t]{2}{*}{ Densité } & Technologie & 0,10330 & 0,03443 & 258,3 & 0,00 \\
\hline & Erreur & 0,00107 & 0,00013 & & \\
\hline
\end{tabular}

\section{Différenciation entre les extraits de fruits de la passion}

Les différents extraits ont été représentés dans le plan F1-F2 (Figure 4). A l'analyse, les deux premières composantes principales expliquent 99,05 $\%$ de la variance totale. De plus, cette représentation montre que les extraits CMFT et JB sont sensiblement proches et forment un groupe différent de RT et de COI. Les extraits RT et COI forment deux groupes nettement distincts. L'analyse du cercle de corrélation a montré que le groupe formé par CMFT et JB est caractérisé par le pH élevé et leur faible densité (Figure 5). Par ailleurs, l'extrait COI est caractérisé par l'acidité, l'ESS, la viscosité, la conductivité et la teneur élevée en vitamine C. En outre, l'extrait RT est caractérisé par sa teneur en flavonoïdes, en polyphénols et par sa turbidité élevée. 


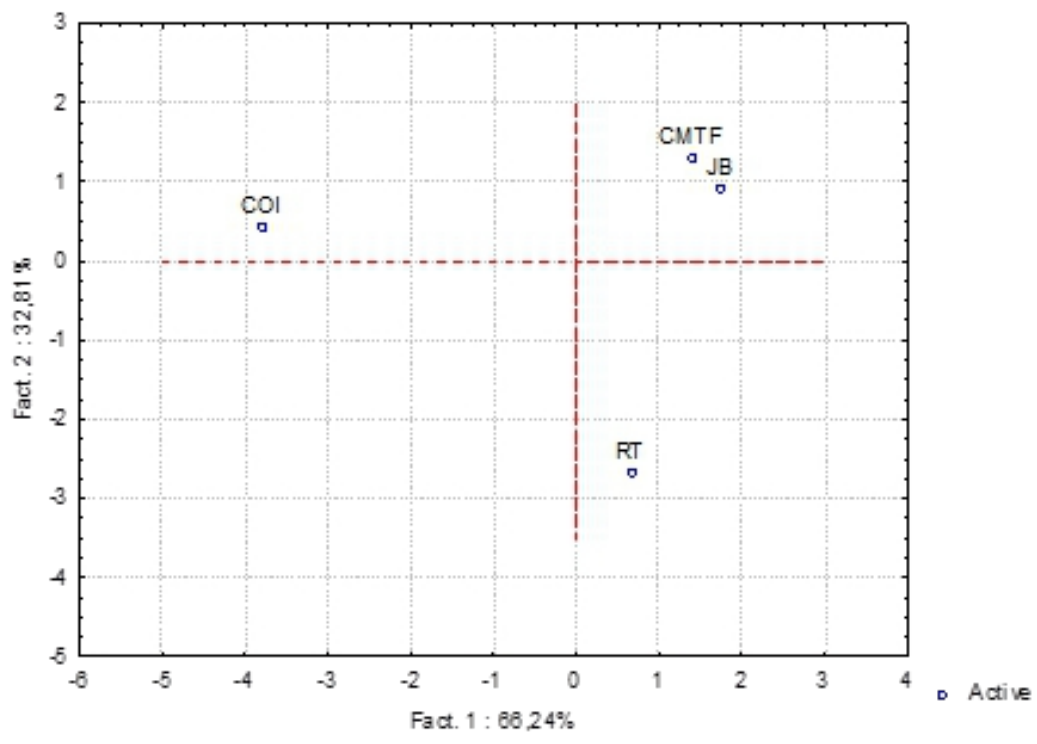

JB : Jus Brut ; CMFT : Jus Clarifié par Microfiltration Tangentielle ;

COI : Jus Concentré par Osmose Inverse ; RT : Retentât.

Figure 4 : Projection des individus dans un plan factoriel F1-F2.

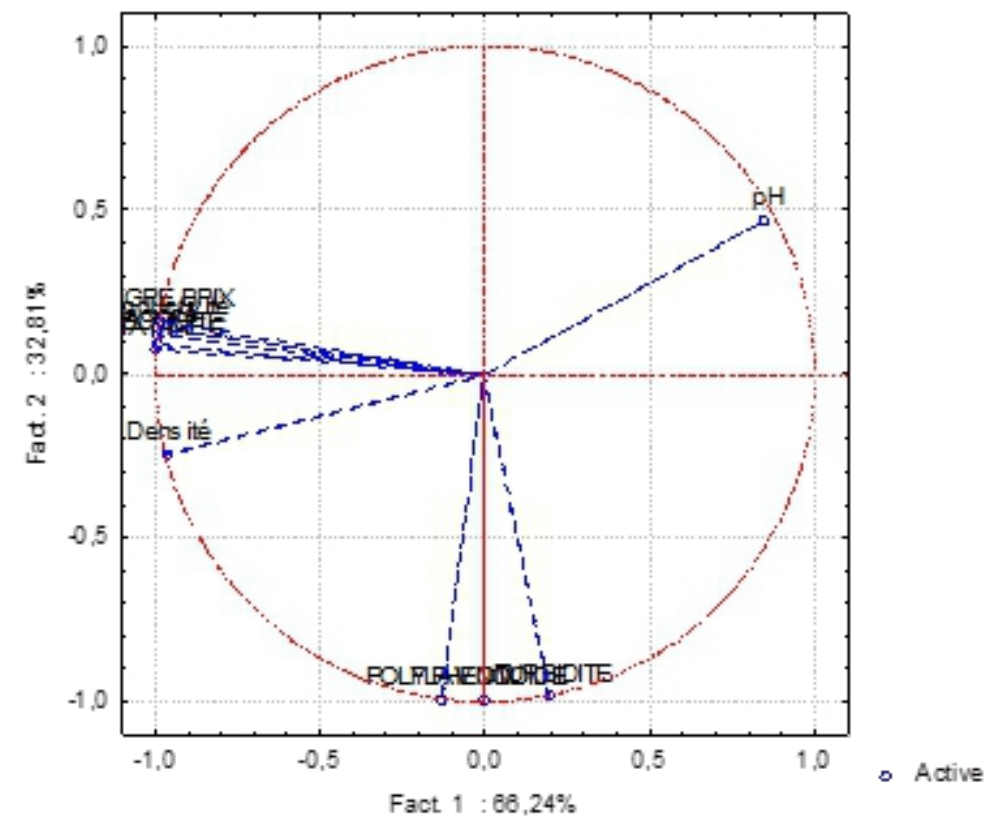

Figure 5 : Cercle de Corrélation des variables dans le plan factoriel F1-F2.

\section{Discussion}

Les conditions opératoires établies pour la fabrication des extraits ont permis d'obtenir un rendement de $89,33 \%$ de jus micro-filtré, un perméat de $16 \mathrm{~L}$ et un concentré de $8 \mathrm{~L}$ avec un FRV de 16,75. Le rendement en jus microfiltré est supérieur à celui de $82 \%$ rapporté par Soro (2012) qui travaillait sur 
la production de jus de pomme de cajou par couplage de procédés membranaires. Ce rendement aurait été meilleur si en plus d'une préfiltration et d'un lavage des membranes, un traitement enzymatique avait été réalisé au préalable (Silva et al., 2005 ; Soro, 2012 ; Pranjal et al., 2014 ; Domingues et al., 2014). En effet, le traitement enzymatique aurait réduit la viscosité du jus, responsable de l'encrassement, et aurait facilité le flux du perméat (Silva et al., 2005). L'encrassement est l'un des facteurs responsables du colmatage de la membrane, induisant un déclin du flux de perméat et donc des performances globales du procédé (Vaillant, 2009). Par ailleurs, les extraits obtenus (CMFT, $\mathrm{JB}$, COI et $\mathrm{RT})$ ont un $\mathrm{pH}$ acide $(\mathrm{pH}<4,5)$. Des résultats similaires ont été rapportés pour les jus de passion (Silva et al., 2005). Ces auteurs ont enregistré des valeurs de l'ordre de 3,10 pour le jus brut et 3,04 pour le jus microfiltré. Le $\mathrm{pH}$ recommandé pour les jus de fruits est inférieur à 4,6 (Codex Alimentarius, 2005). Les valeurs de $\mathrm{pH}$ des différents extraits analysés sont conformes à cette norme. En outre, la teneur en polyphénols des extraits de passion produits est inférieure à celle de l'ordre de $991 \mathrm{mg} / 100 \mathrm{~g}$ Eq.AG rapportée par Soro (2012) dans la clarification du jus de pomme de cajou. De même, la teneur en vitamine $\mathrm{C}$ des extraits de passion est inférieure à celle des extraits de pomme de cajou qui est de $2059 \pm 8 \mathrm{mg} / \mathrm{kg}$ (Soro, 2012).

La corrélation positive et significative existant entre la conductivité et l'acidité titrable s'explique par le fait que plus la solution est acide, plus elle conduit le courant électrique (Guillobez et Arnaud, 1985). De plus, Elgamouz (2016) lors du suivi de la teneur en vitamine $\mathrm{C}$ dans des jus de fruits industriels a montré que plus la teneur en vitamine $\mathrm{C}$ augmente, plus l'acidité est élevé. Ceci confirme bien la corrélation significative observée entre la vitamine $\mathrm{C}$ et l'acidité des extraits produits dans la présente étude. Les extraits de fruits de la passion sont donc caractérisés par leur acidité, leur conductivité et leur teneur en vitamine C. Malgré l'existence de corrélations significatives entre les paramètres caractérisant les extraits de fruits de passion produits, l'analyse de variance multidimensionnelle (MANOVA) a révélé une différence significative entre ces extraits en tenant compte de l'ensemble des paramètres. Dès lors, le procédé de clarification-concentration a un effet significatif sur ces paramètres physicochimiques. D'ailleurs, l'ACP a confirmé l'existence de différences entre les extraits étudiés. A ce propos, les caractéristiques physicochimiques diffèrent significativement d'un type d'extrait à un autre. Ainsi, l'analyse comparée du jus brut et du jus microfiltré a-t-elle révélé une diminution de la turbidité de 99,18\%. Des résultats similaires ont été rapportés dans la microfiltration de jus de fruits (Silva et al., 2005 ; Cardoso et al., 2012 ; Soro, 2012). Cette évolution de la turbidité justifie de la performance du dispositif de clarification. La microfiltration a donc permis l'élimination complète de la pulpe en suspension dans le perméat de jus ; ce qui a donné lieu à un jus clarifié. Ce procédé a affecté l'ensemble des paramètres excepté la 
viscosité du jus brut. Par ailleurs, la microfiltration n'a pas influencé la viscosité certainement à cause de l'absence de traitement enzymatique préalable du jus brut (Silva et al., 2005 ; Liberto et al., 2016).

En outre, les extraits de fruits de la passion produits renferment des métabolites secondaires tels que les flavonoïdes et les polyphénols totaux. Ces composés sont d'excellents antioxydants capables de renforcer les défenses des consommateurs contre le stress oxydatif (Cissé et al., 2009). Aussi, par leur propriété de piégeurs de radicaux libres, les flavonoïdes sont impliqués dans la prévention des dommages oxydatifs causés par les espèces oxygénées activées sur les molécules cellulaires (Chang et al., 2006). Par conséquent, les extraits de fruits de la passion pourraient être recommandés dans la prévention de diverses maladies (Asthme, hypertension, maladies articulaires dégénératives) (Anonyme, 2010). En addition, les concentrations en polyphénols totaux et flavonoïdes diminuent au cours de la production d'extraits clarifiés de fruits de la passion. Ce résultat confirme bien celui rapporté par Soro (2012) qui travaillait sur la clarification jus de pomme de cajou. Soro (2012) présente ce phénomène comme étant bénéfique et souhaitable dans la mesure où il réduit les composés responsables de l'astringence (polyphénols totaux) des pommes de cajou.

Par ailleurs, lors du processus de clarification-concentration par procédés membranaires, les teneurs des différents composés dosés dans les extraits microfiltrés sont inférieures à celles des extraits concentrés avec un facteur de réduction volumique égale à 16,75. Cette observation a été déjà faite lors de la clarification-concentration des extraits de Delonix regia et de Tectona grandis (Adjé et al., 2012 ; Koffi et al., 2015). À la fin de l'opération de clarification-concentration, les extraits concentrés contiennent une quantité de composés multipliée par des facteurs de concentration variant entre 2,15 à 9,39. Ainsi, les techniques séparatives membranaires (MFT/OI) en réduisant le volume du jus de passion microfiltré permettent de concentrer les biomolécules présentes (Koffi et al., 2015). Ceci explique l'augmentation de la teneur de certains paramètres dont l'acidité, observée après le passage à l'osmose inverse. La réduction volumique des extraits par osmose inverse entrainerait donc une concentration des acides organiques présents. Aussi, cette réduction permet d'expliquer l'augmentation des teneurs en flavonoïdes, en polyphénols totaux et en vitamine $\mathrm{C}$ de l'extrait concentré. Dès lors, l'activité antioxydante de l'extrait concentré s'en trouve améliorer par rapport à d'autres extraits. En outre, de même que le jus brut, le retentât possède des éléments nutritifs. Pour l'industrie des jus de fruits, le retentât ne doit pas être considéré comme un déchet. En effet, il peut à nouveau être recombiné à du jus clarifié après pasteurisation thermique ou mélangé avec du jus brut. Toutefois, le taux optimal de retentât à ajouter au perméat doit être étudié (Vaillant et al., 1999). 


\section{Conclusion}

Le but visé a été d'appliquer les technologies membranaires telles que la microfiltration tangentielle et l'osmose inverse à la production d'extraits de fruits de passion en vue d'en déterminer l'impact sur les caractéristiques physicochimiques des extraits de fruits de passion. Il ressort que les procédés membranaires MFT-OI ont un effet significatif $(\mathrm{p}<0,05)$ sur les caractéristiques physicochimiques des différents extraits de passion. Les paramètres physicochimiques significativement influencés sont le $\mathrm{pH}$, la densité, l'ESS, l'acidité, la viscosité, la teneur en vitamine $\mathrm{C}$, les teneurs en flavonoïdes et en polyphénols. Par ailleurs, la microfiltration tangentielle a permis de clarifier l'extrait brut de passion en éliminant pratiquement tous les éléments en suspension avec un pourcentage de 99,18. De plus, l'extrait microfiltré a été concentré par osmose inverse avec un facteur de réduction volumique de 16,75. Cela a favorisé la concentration des composés tels que la vitamine $C$ (2,15 fois), les polyphénols (3,30 fois) et les flavonoïdes (7,85 fois). L'extrait concentré et stabilisé peut être utilisé comme base/ou ingrédient pour la fabrication de produits divers.

\section{References:}

1. Adjé A. F., Lozano F. Y., Guernevé L. C., Lozano R. P., Meudec E., Adima A. A. \& Gaydou E. (2012). Phenolic acid and flavonol water extracts of Delonix regia red flowers. Industrial Crop and Products, 37: 303-310.

2. Adjé A. F., Niamke B. F. \& Adima A. A. (2013). Couplage de technologies membranaires pour la production d'extraits stables de bissap (Hibiscus sabdariffa L., Malvaceae). AGRAR: L'agriculture face aux défis de l'alimentation et de la nutrition en Afrique : quels apports de la recherche dans les pays cotonniers (pp 589-602), 4-6 Juin 2013, Institut National Polytechnique Houphouët-Boigny, Yamoussoukro, Côte d'Ivoire.

3. Anonyme (2010). Fichier canadien sur les éléments nutritifs, Santé Canada.

4. AOAC (2000). Official Methods of Analysis (17th edn). Association of Official Analytical Chemist: Washington, D C.

5. Araya-Farias M. (1999). La clarification du jus de pomme par électroflottation. Mémoire l'obtention du grade de Maître ès sciences, Faculté des sciences de l'agriculture et de l'alimentation, Université Laval, Canada.

6. Barnes J., Anderson L. A. \& Phillison J. D. (2002). Herbal medicines. Second Edition, Pharmaceutical Press, Londres, Angleterre.

7. Boko A. C. E. (2018). Application des technologies membranaires à la production d'extraits de grenadille (Passiflora edulis). Mémoire de 
Master de Génies des procédés, Université Jean Lorougnon Guédé, Daloa (Côte d'Ivoire).

8. Boutarfa M. (2012). Caractérisation physique et rhéologique de la bentonite de maghnia. Mémoire en vue de l'obtention du diplôme de magister, Option : rhéologie et transport des fluides complexes, Université des sciences et de la technologie d'Oran Mohamed Boudiaf, Algérie.

9. Cardoso D. O. R., Docê R. C. \& Sueli T. B. D. (2012). Clarification of passion fruit juice by microfiltration: Analyses of operating parameters, study of membrane fouling and juice quality. Journal of Food Engineering, 111: 432-439.

10. Chang Y. C., Huang K. X., Huang A. C., Ho Y. C. \& Wang C. J. (2006). Hibiscus anthocyanins-rich extract inhibited LDL oxidation and oxLDL-mediated macrophages apoptosis. Food and Chemical Toxicology, 44(7): 1015-1023.

11. Cissé M., Dornier M., Sakho M., Ndiaye A., Reynes M. \& Sock O. (2009). Le bissap (Hibiscus sabdariffa L.) : composition et principales utilisations. Fruits, 64(3) : 179-193.

12. Cissé M., Soro D. \& Dornier M. (2014). Jus de fruits tropicaux et techniques de filtration membranaires. Congrès AFTER: Favoriser l'accès au marché des produits traditionnels africains (pp 32-33), 1112 novembre 2014, Dakar, Sénégal.

13. Codex Alimentarius (2005). Programme Mixte Fao/Oms sur les normes alimentaires. FAO, Rome (Italie).

14. Domingues R. C. C., Fara J. B., Silva B. R., Cardoso V. L. \& Reis M. H. (2012). Clarification of passion fruit juice with chitosan: effects of coagulation process variables and comparison with centrifugation and enzymatic treatments. Process Biochemistry, 47: 467-471.

15. Domingues R. C. C., Ramos. A.A., Cardoso L.V. \& Reis M. H. (2014). Microfiltration of passion fruit juice using hollow fibre membranes and evaluation of fouling mechanisms. Journal of Food Engineering, 121: 73-79.

16. Elgamouz S. (2016). Le suivi de la teneur de la vitamine $C$ dans un jus industriel. Mémoire de Master, Sciences et Techniques: CMBA chimie des molécules bioactives, de l'Université Sidi Mohammed Ben Abdellah, Maroc.

17. Guillobez S. \& Arnaud M. (1985). Bilan ionique en solution : Lien avec le $\mathrm{pH}$ et la conductivité électrique, Aide au classement des horizons de sol. L'Agronomie Tropicale, 40 (3) : 246-252.

18. Haendler L. (1965). La passiflore sa composition chimique et ses possibilités de transformation. Fruits, 20 (5) : 235-245. 
19. Hoff M. \& Cremers G. (2005). Le Jardin Guyanais Inventaire des plantes cultivées et des adventices des jardins de Guyane française. Journal de Botanique de la Société de Botanique, 29 : 3-40.

20. Koffi E. N., Meudec E., Adjé F. A., Lozano P. R., Lozano Y. F. \& Bekro Y. A. (2015). Effect of reverse osmosis concentration coupled with drying processes on polyphenols and antioxidant activity obtained from Tectona grandis leaf aqueous extracts. Journal of Applied Research on Medicinal and Aromatic Plants, 2: 54-59.

21. Liberto A., Fernández J. M. \& Chaparo L. (2016). Evaluación de una bebida a base de jugo de parchita (Passiflora edulis var. flavicarpa) clarificada por microfiltración tangencia. Revista Agrollanía, 13 :1821.

22. Marinova D., Ribarova F. \& Antanassova M. (2005). Total phenolics in bulgarian fruits and vegetables. Journal of the University of Chemical Technology and Metallurgy, 40(3): 255-260.

23. Pranjal S., Deshmukh M. S. S. \& Raju P. S. (2014). Influence of moisture content on the thermophysical properties of the enzyme clarified sapota (Achras sapota L.) juice. International Food Research Journal, 21 (4): 1539-1552.

24. Silva T.T., Modesta R. C.D., Penha E. M., Matta D.V. \& Cabral L. M. C. (2005). Suco de maracujá orgânico processado por microfiltração. Pesquia Agropecuária Brasileira, 40(4) : 1678-3921.

25. Soro D. (2012). Couplage de procédés membranaires pour la clarification et la concentration du jus de pomme de cajou : performances et impacts sur la qualité des produits. Thèse de doctorat en Génie des Procédés, Université Montpellier SupAgro, France.

26. Vaillant F. (2009). Procédés de valorisation agroalimentaire des fruits tropicaux et impacts sur la qualité. Habilitation à diriger de recherches. Ecole doctorale science de procédés, Université Montpellier II, France.

27. Vaillant F., Millan P., O’Brien G., Dornier M., Decloux M. \& Reynes M. (1999). Crossflow microfiltration of passion fruit juice after partial enzymatic liquefaction. Journal of Food Engineering, 42 (4): 215-224.

28. Winston D. \& Kuhn M. A. (2000). Herbal Therapy \& Supplements. Lippincott, Philadelphia, États-Unis.

29. Wood J. E., Senthilmohan S. T. \& Peskin A. V. (2002). Antioxidant activity of procyanidin-containing plant extracts at different pHs. Food Chemistry, 77: 155-161. 